\title{
Fusion for medical image based on discrete wavelet transform coefficient
}

\author{
Zahraa Yaseen Hasan ${ }^{1}$, Rusul H. Al_taie ${ }^{2}$, Hawraa abd Al_kadum hassan ${ }^{3}$ \\ ${ }^{1}$ College of science for women, University of Babylon, Iraq \\ ${ }^{2}$ College of Arts, University of Babylon, Iraq \\ ${ }^{3}$ College of information technology, University of Babylon, Iraq
}

\begin{tabular}{l}
\hline Article Info \\
\hline Article history: \\
Received May 15, 2020 \\
Revised Aug 30, 2020 \\
Accepted Oct 1, 2020 \\
\hline
\end{tabular}

\section{Keywords:}

Discrete wavelet transform

Image fusion

Medical image

Peak signal to noise ratio

Signal to noise ratio

\begin{abstract}
More recent digital camera introduced enormous facilities for users from different specifications to take images easily, but user still wants to improve these images, which contain different problems as ambiguous and unclear colors, because cloudy weather, bright light, dark region and it's taken from remote distances. This paper aims to use a new approach for fusion images by using a wavelet coefficient based on PSNR and SNR measures (the technical result) instead of using the max, min, average values. Wavelet coefficient of each sub band is compared between them, sub band had a value higher is selected for fusion. Firstly, discrete transform applied to the medical images with 2level. Then, PSNR ratio and SNR measures computed for each sub-band. After that PSNR and SNR values have been compared for each sub-band to opposite sub-band and it selected the better value of measures. Secondly, PSNR and SNR values gathered for each image. Then select the image that contains higher PSNR and lower SNR values for purpose fusion. Finally, perform an inverse discrete wavelet to fused image to transform the image to the spatial domain. The results of the work showed that the wavelet coefficient used to preserve the image details and removed the noise. PSNR of 1level is higher than 2level. This paper makes the image more clearer and informative than original images.
\end{abstract}

This is an open access article under the CC BY-SA license.

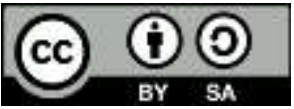

\section{Corresponding Author:}

Rusul H. Al_taie

College of Arts, University of Babylon

Hilla, Iraq

Email: rusul.jasem@uobabylon.edu.iq

\section{INTRODUCTION}

At present, image fusion is considered as one of the kinds of integrated technology information, it has played a major role in several domains and production of high-quality images. In the recent years increase the digital image usage because the technology development and devices that operate with images, classic cameras depend on physical principal while digital cameras operate on convert the light into electrical charge and the image is converted into a series of zeros and ones to represent pixel value in the image. In the case, the image is taken from the cloudy weather or dark region that lead to problem happen in the image details is more a problem that happens to the users in work them like medical images dark, images are taken from a satellite, etc. $[1,2]$. Also, The images are degraded by different types of noise and blur. This paper introduces image fusion based on the discrete wavelet coefficient to enhance the image based on the best value of the measure. This paper aims to use the wavelet coefficient with a fusion method based on PSNR and SNR value, the wavelet coefficient for each sub band is compared with other sub band and is selected 
better value of the metrics. A fused image is formed based on the optimal sub-band that has a high PSNR value from each image.

Discrete wavelet transform is one of the image fusion methods. It considers a good tool and helpful for fusion and it is used in many applications like speech recognition, fractal recognition, image denoising, and image compression. The input image is decomposed by using a discrete wavelet transform into approximation and detail coefficients based on low pass filter and high pass filter. Figure 1 shows applying DWT method on the input images. A low pass filter produces approximation coefficients, while the high pass filter produces detailed coefficients $[3,4]$.

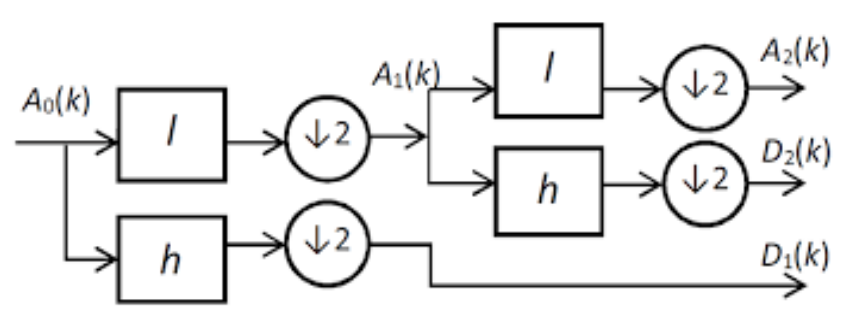

Figure 1. 2level discrete wavelet transform [5]

The Discrete Wavelet transform is a domain transform method. It is a more common method used for image fusion. Discrete wavelet transforms decomposes the original images into four sub-bands by the filters with each level K, called approximation coefficients, vertical details, horizontal details, and diagonal details. The processing wavelet coefficients of each level are the input of the next level [6, 7]. The fused image is gained by utilizing the IDWT process. Wavelet transform allows for analysis of the images by using different levels of accuracy. Discrete wavelet transform tends for choosing the distinguishing features of an image. The wavelet transform is utilized in different areas such as image fusion, texture analysis, feature detection, data compression, etc. A discrete transform based fusion scheme as shown in Figure 2 [8, 9].

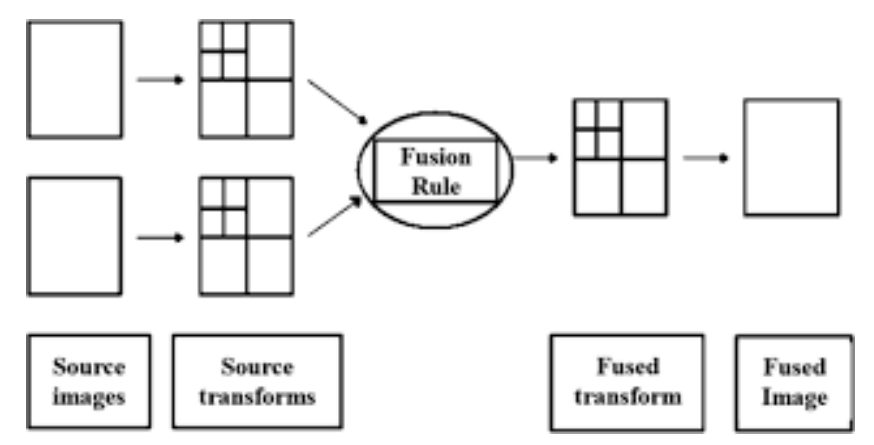

Figure 2. Diagram of discrete transform based image fusion [6]

In many situations, a single image cannot describe the scene accurately, because the scene is usually taken using more than one sensor. Only a single image is more appropriate for human or machine processing, therefore it is significant to fuse all the images from different sensors to form a single image with all relevant information. With the spreading of image processing, image fusion has been a significant subject in several associated areas such as computer vision, remote sensing, object detection, medical imaging, image classification [10]. In general, term fusion is a method extracting information from different domains in Figure 2. The goal of fusion is merging associated information from two or more of data to procedure data that is more precise than the single data.

Image fusion is the method of mixing the information from a group of images to produce an image that is more informative than the input images. The main objective of fusion is to increase the spatial resolution of the original image and improving the image characteristics to make more clarification $[5,6]$. Image fusion can be defined as a manner of mixing the information from a set of images to form one image 
and produce a fused image that is more complete and informative than the original images [11, 12]. The purpose of image fusion is to construct a fused image output that supplies the information which is more reliable and more suitable. The general definition of image fusion is the blending of two or more various images to form a new image by implementing a suitable algorithm as in Figure 3. A good fusion is getting on information from the original images and the resulted image without any changeability. The fusion algorithm must satisfy the following requirements:

a) It keeps on important features of the original image without loss of detail in the output image.

b) It avoids any artifacts in the image as the noise [13].

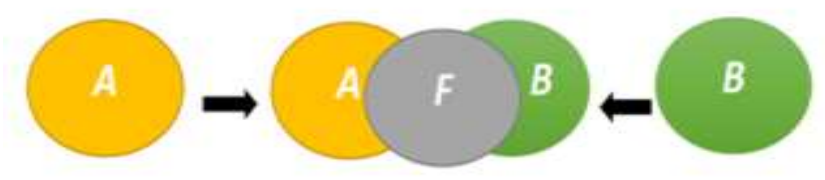

Figure 3. Graphical representation of fusion [11]

The fusion process can apply at various levels of information representation is signal level, pixellevel, and feature level. Signal level fusion merges a set of signals that have the identical general design. At pixel level (image-level) combines sets of pixels of images to make the fusion decision like maximum, minimum, and average pixels. While in fusion based on the feature level, the information will be extracted from source images individually and then merged based on original image features. In fusion based on the decision level, the information will extract from each source image individually and then the decisions are made for each original channel. These decisions are joined to produce the final decision [11-14].

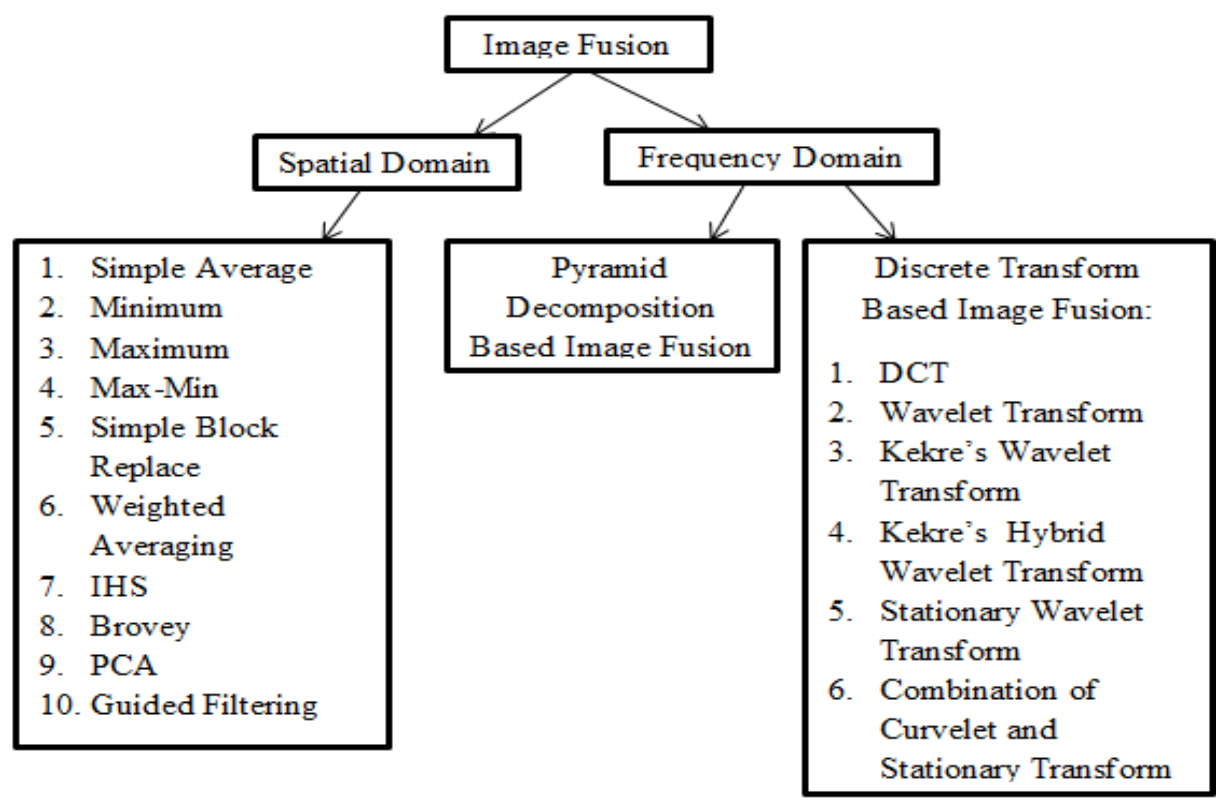

Figure 4. Image fusion techniques [10]

The fusion process is applied based on characteristics of a set of regions of input images, such as contrast, shape, and texture. The fusion process is applied in the spatial and frequency domain is shown in Figure 4 [10].

Image fusion can be distinguished by the following categories:

a) Multi view fusion: Images are the same modal and time, but in various conditions are shown in Figure 5. 
b) Multimodal fusion: Images are different modalities: MRI, visible, infrared, ultraviolet, etc. as shown in Figure 6.

c) Multi temporal fusion: images are taken at various times, but with the same modality are shown in Figure 7.

d) Multi focus fusion: images of a 3D scene will be taken frequently with different central lengths as shown in Figure 8.

e) Fusion for image deconvolution (restoration): Where each image involves the true region and degradation region, which removes the degradation part by using the fusion process is shown in Figure $9[5,11]$.

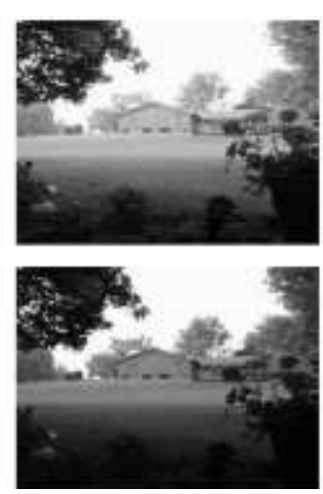

\section{Foreground}

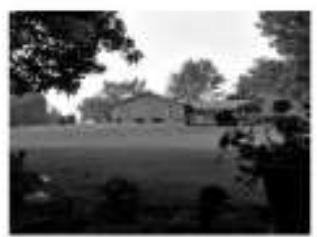

\section{Background}

Figure 5. Multi view image fusion [11]
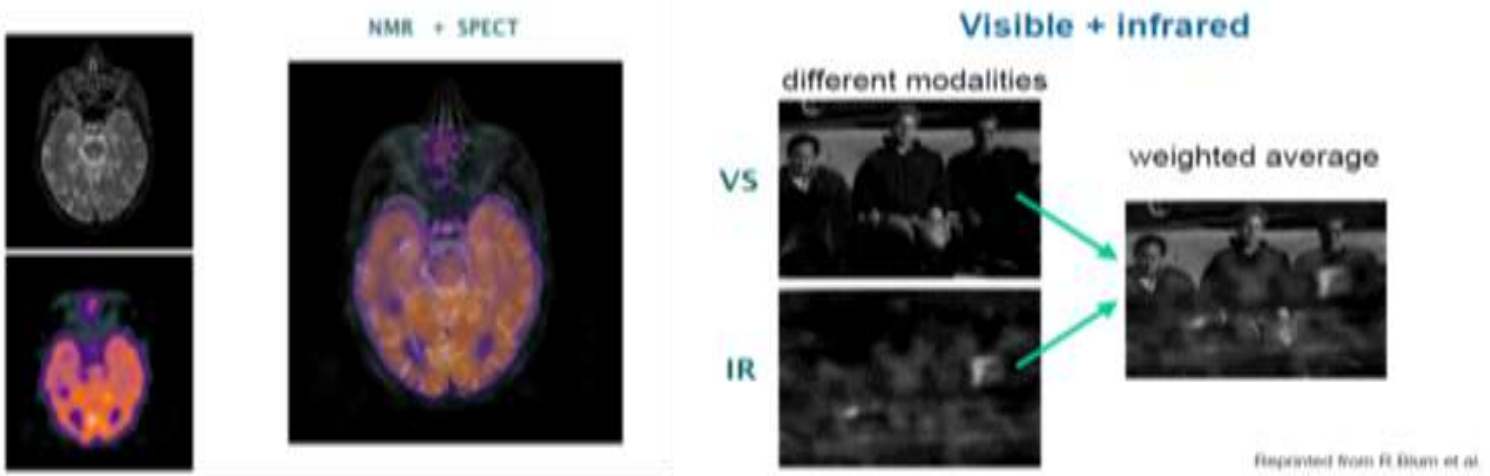

Figure 6. Multimodal image fusion [11]

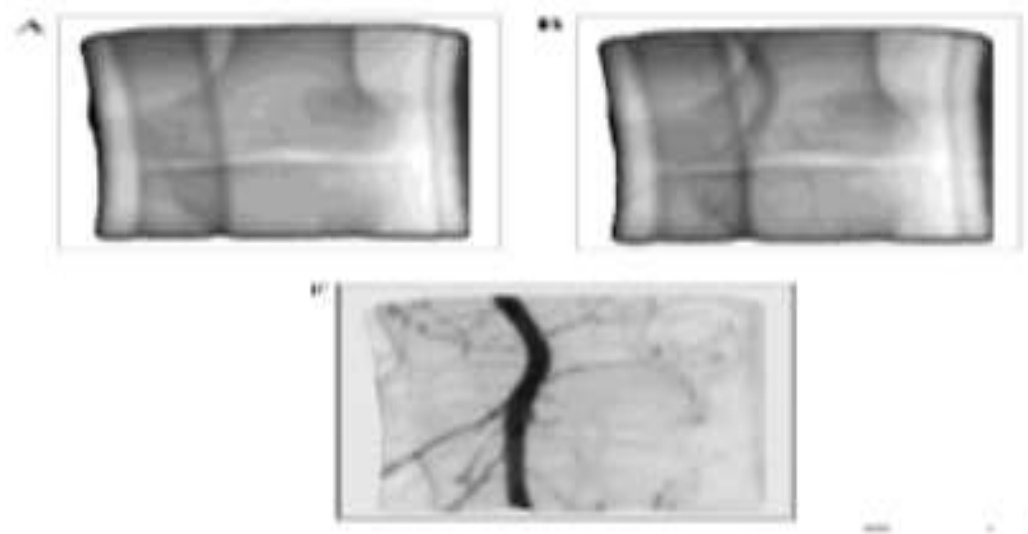

Figure 7. Multi temporal image fusion [11] 


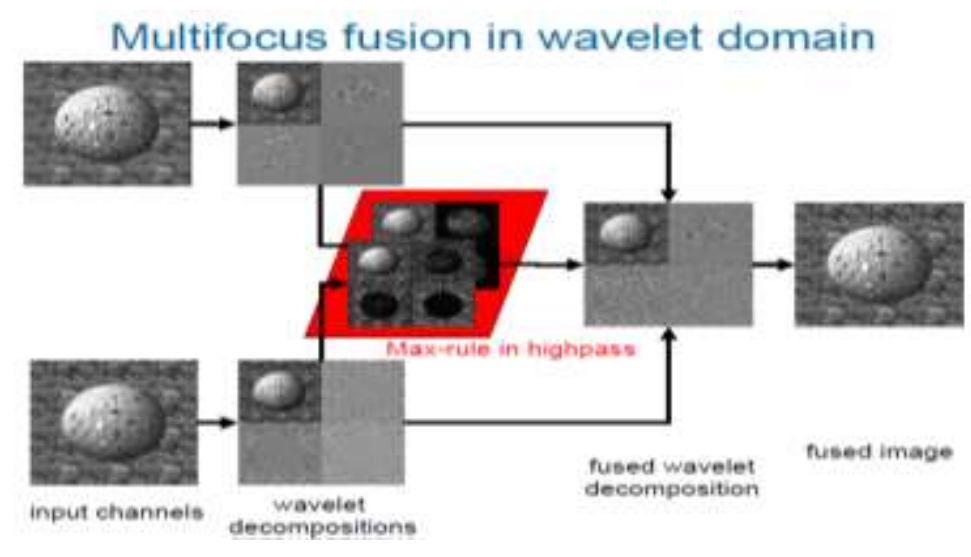

Figure 8. Multi focus image fusion [11]

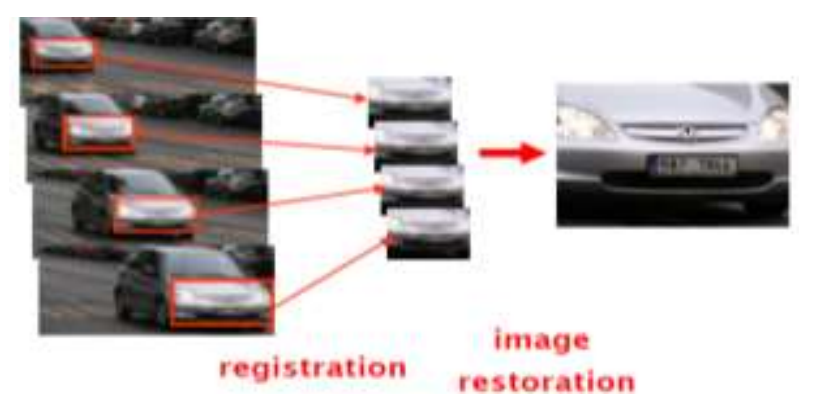

Figure 9. Image restoration for fusion [11]

\section{RELATED WORK}

Hari et al. [15] presented image fusion using the wavelet transform. They used the wavelet method and several rules of fusion (minimum, maximum, and average) on the MRI and CT images. They show that a good way that gives the smallest MSE and largest PSNR values.

Image fusion based on wavelet proposed by Laxman et al... They performed a wavelet method and weighted that gain a good fusion of CT/PET images compare to CT or PET separately [16]. The paper of [17] presented image fusion for CT/PET based on wavelet transform. He performed different rules of fusion. He showed contrast rule provides the best.

Ram Nivas et al. [18] presented a fusion of medical images based on the wavelet transform. They utilized the wavelet transform, PCA for fusing. They exhibited DWT enhances the edge and Bicubic interpolation enhances the resolution. Efficiency MRI and CT images were presented by Prerana and Deepali. They extended this method based on curvelet transform with wavelet transform methods to attain improving the performance [19]. Nayera and Deep [20] presented medical image fusion with DWT. They performed various rules of fusion, containing max, min, average, and min max for medical image fusion.

The above methods were used fusion method with DWT based on the max, min, average value of wavelet coefficient while in this paper can be used the coefficient of wavelet for comparing each sub band with the same sub band of another image based on the best value of PSNR and SNR. The sub band has a better value of the measure is selected for fusion to form the fused image.

\section{MATERIALS AND METHODS}

This paper uses the coefficient of wavelet to compare each sub band with the same sub band of other image based on the best value of PSNR and SNR measures. The sub band contains on better value of the measure is chosen for fusion and form the output fused image.

This paper presented a good method for image fusion by using discrete wavelet transform coefficients. The medical image is used in this paper. The main idea is to apply the DWT to the images. After that PSNR and SNR are computed for each sub band and combine the sub-band that has a better value of the measure. Then inverse discrete wavelet transform is performed to convert the fused image from the frequency domain to the spatial domain. Figure 10 presented the proposed method that contains a set of steps: 


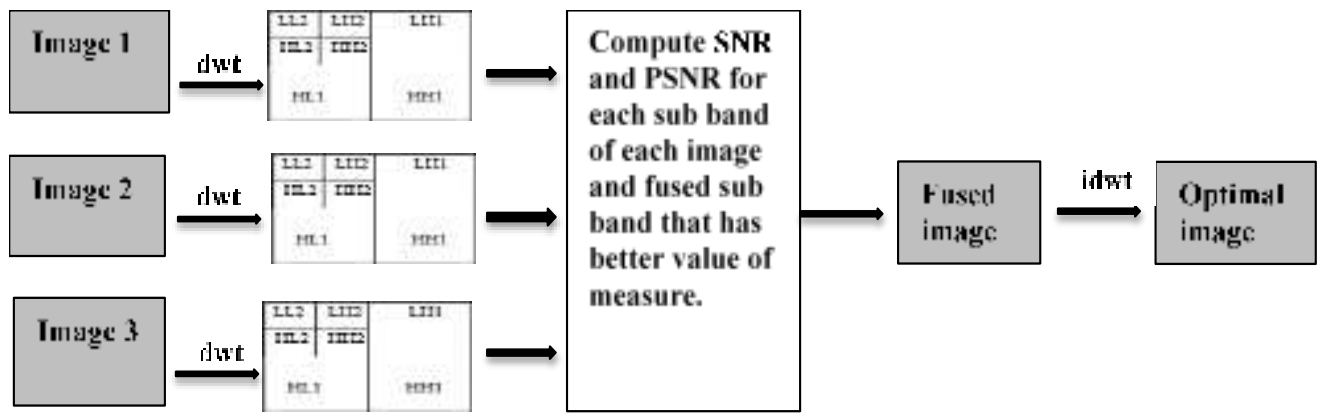

Figure 10. Block diagram of the proposed method

\subsection{Select set of images}

The images are selected in this proposed are medical images that are converted to two dimensions array. The images that are performed must be the same size and same scene.

\subsection{Apply discrete wavelet transform on images}

Two-level wavelet transform is performed on medical images and consistent set of sub-bands (vertical, horizontal, and diagonal coefficients for each level), see Figure 1. The medical input image is decomposed by using a DWT into approximation coefficients and detail coefficients based on low pass filter and high pass filter. DWT coefficients on the images are computed by using the low pass and high pass filters according to (1) and (2).

$$
\begin{aligned}
& \operatorname{app}(r, c)=\sum_{k} i m(r, l) g i(c-l) \\
& \operatorname{det}(r, c)=\sum_{k} \operatorname{im}(r, l) h i(c-l)
\end{aligned}
$$

Where im is the input image, gi and hi represent low pass filter and high pass filter respectively.

\subsection{Find PSNR and SNR values for sub-bands}

PSNR and SNR values are computed for sub-bands according to (4) and (5).

\subsection{Fusion process}

In this step PSNR value is gathered for each sub band and then is fused the optimal sub-band has value large. The optimal image is selected based on the highest PSNR value.

\subsection{Perform inverse discrete wavelet transform}

Fused imaged is in the frequency domain. Apply IDWT to transform it from the frequency to the spatial domain. IDWT is calculated according to the low and high pass filter DWT process, but in a reverse manner to result in the output image (optimal image).

\section{IMAGE QUALITY METRICS}

Image quality can be defined as a feature of an image that processes the image corrupted compared to a pure image. Performance metrics are used essentially to measure the possible benefits of fusion and also used to compare results gained using various algorithms [21].

\subsection{Mean square error (MSE)}

It is used to measure the differences between the values of the input image and fused image divided by the dimensions of the image according to (3). The value of MSE must be as small as possible [22-24].

$$
\mathrm{MSE}=\sum_{i=0}^{n-1} \sum_{j=0}^{m-1} \frac{(s(i, j)-f(i, j)) 2}{n * m}
$$

Where $n$ and $m$ are the dimensions of the image, $s(i, j)$ is the pixel values of the original image. $f(i, j)$ is the pixel values of the resulted image. 


\subsection{Peak signal to noise ratio (PSNR)}

It is the ratio between the maximum possible power of an image and the power of corrupting noise that affects the fidelity of its representation. PSNR calculates from the following Equation:

$$
\mathrm{PSNR}=10 * \log _{10}\left(\frac{255^{2}}{M S E}\right)
$$

So that computing PSNR should calculate the mean square error (MSE) in the beginning. The value of PSNR must be as large as possible [25].

\subsection{Signal to noise ratio (SNR)}

The signal is the information content of the data of the original MS image MK, while the merging FK can cause the noise, like an error that is added to the signal. The RMSK of the signal to noise ratio can be used to calculate the signal to noise ratio SNRK, given by:

$$
\mathrm{SNR}_{\mathrm{k}}=\sqrt{\frac{\sum_{i}^{m} \sum_{j}^{n} F(i, j)^{2}}{\sum_{i}^{m} \sum_{j}^{n}\left(F_{k(i, j)-M_{k(i, j))}}{ }^{2}\right.}}
$$

Where FK is the fused pixel and MK is the original pixel [25-27].

\section{RESULTS}

The results are applied to the medical images as is given in Figure 11. The earlier studies were used fusion method with DWT based on the max, min, average value of the wavelet coefficient. The key idea of this paper is on getting the image clear, complete, and more informative than input images using a fusion based on the best value of measures. After DWT is applied to the images, PSNR and SNR for each sub band are compared to other sub brands of another image, the sub band that has a better value of the measure is selecting for the fusion process. The inverse of DWT is computed to get on the fused image (output image). Figure 11 explains applying the fusion technique on the medical images to compose the fused image. Fused image results from images merging based on the higher PSNR value that sub-band of images has large PANR value. Because the fused image in the frequency domain is applied inverse discrete wavelet to transform it into the spatial domain.

Figure 12 clarifies using the fusion method on input medical images to form the fused image. The fused image is computed from the sub band of images merging based on the better measure value of each sub band, sub-band of images has large PANR value is selected for fusion. The inverse wavelet transform was applied to the fused image to change the image from frequency domain into spatial domain.

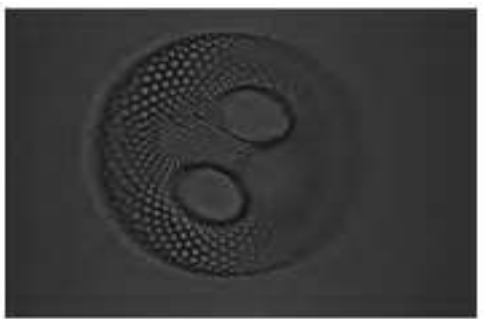

a) Input medical image

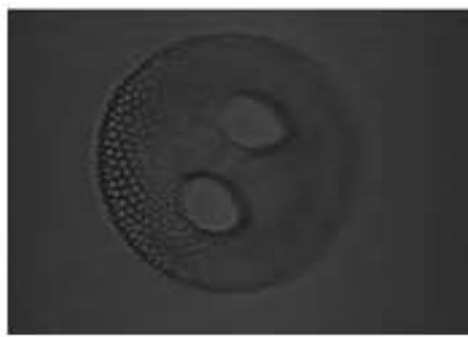

c) Input medical image

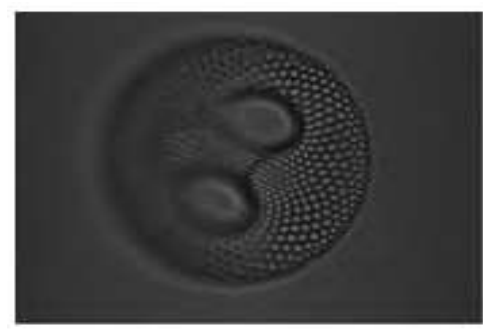

b) Input medical image

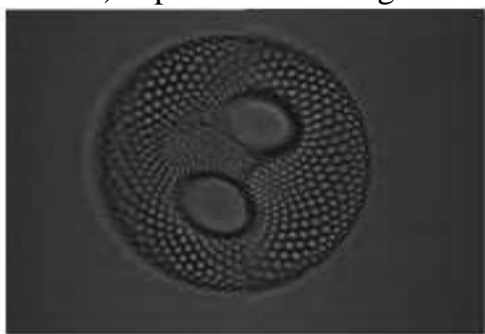

d) Fused image

Figure 11. Original medical images and fused image 
Table 1 displays the PSNR and SNR measures for each sub band of different images in Figure 11 while Table 2 illustrates the sum of PSNR and SNR values of each image, sub bands of each image have a better value of the measure is the selected to form the optimal image.

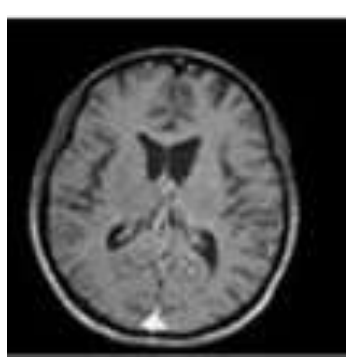

a) Input image

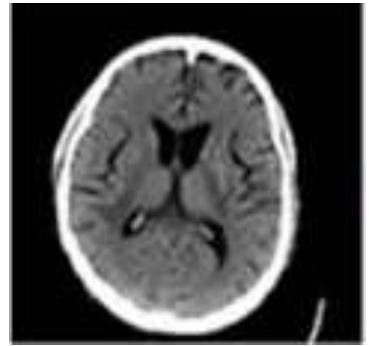

b) Input image

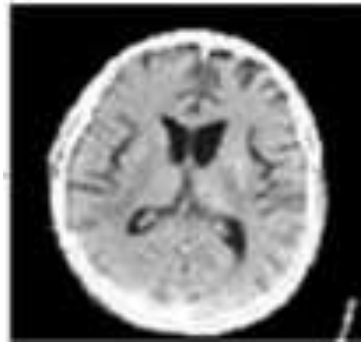

c) Input image

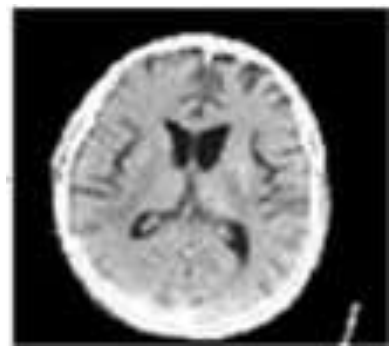

d) Fused image

Figure 12. Input images and fused image

Table 1. PSNR and SNR value of each sub band of medical images (400*400)

\begin{tabular}{lcc}
\hline Sub bands of images & Peak Signal to Noise Ratio value & Signal to Noise Ratio value \\
\hline LL2 of image 1 and 2 & 20.288 & 0.007 \\
LL2 of image 1 and 3 & 20.034 & 0.010 \\
LL2 of image 2 and 3 & 23.878 & 0.005 \\
LH2 of image 1 and 2 & 23.188 & 0.021 \\
LH2 of image 1 and 3 & 48.130 & 0.022 \\
LH2 of image 2 and 3 & 23.248 & 0.009 \\
HL2 of image 1 and 2 & 22.085 & 0.022 \\
HL2 of image 1 and 3 & 31.334 & 0.017 \\
HL2 of image 2 and 3 & 21.883 & 0.011 \\
HH2 of image 1 and 2 & 25.923 & 0.007 \\
HH2 of image 1 and 3 & 48.130 & 0.031 \\
HH2 of image 2 and 3 & 25.983 & 0.062 \\
LH1 of image 1 and 2 & 29.943 & 0.010 \\
LH1 of image 1 and 3 & 32.196 & 0.011 \\
LH1 of image 2 and 3 & 32.030 & 0.010 \\
HL1 of image 1 and 2 & 27.215 & 0.008 \\
HL1 of image 1 and 3 & 24.561 & 0.017 \\
HL1 of image 2 and 3 & 27.331 & 0.022 \\
HH1 of image 1 and 2 & 38.070 & 0.020 \\
HH1 of image 1 and 3 & 40.526 & 0.018 \\
HH1 of image 2 and 3 & 39.850 & 0.032 \\
\hline
\end{tabular}

Table 2. Sum of PSNR and SNR value of each sub band

\begin{tabular}{ccccc}
\hline No. of Level & No. of Image & Sub band & Peak Signal to Noise Ratio value & Signal to Noise Ratio value \\
\hline \multirow{4}{*}{ Image 1 } & LH & 62.140 & 0.053 \\
& & HL & 51.777 & 0.051 \\
& & HH & 78.596 & 0.104 \\
& Image 2 & LH & 61.973 & 0.053 \\
& & HL & 54.546 & 0.052 \\
& & HH & 77.921 & 0.081 \\
& & LH & 64.227 & 0.070 \\
& Image 3 & HL & 51.893 & 0.039 \\
& & HH & 80.377 & 0.048 \\
& & LH & 71.319 & 0.030 \\
& & HL & 53.420 & 0.041 \\
& Image 1 & HH & 74.054 & 0.063 \\
& & LL & 40.322 & 0.032 \\
& & LH & 46.436 & 0.045 \\
& & HL & 43.969 & 0.027 \\
& Image 2 & HH & 51.907 & 0.050 \\
& & LL & 44.166 & 0.048 \\
& & LH & 71.379 & 0.045 \\
& & HL & 53.217 & 0.027 \\
& Image 3 & HH & 74.114 & 0.050 \\
& & LL & 43.912 & 0.071 \\
\hline
\end{tabular}




\section{CONCLUSIONS}

This paper proposed merging medical images based on the wavelet coefficient. The results of this application are given that the image has a higher PSNR value is the better. A fused image is formed based on combining a set of sub bands of different images that have better PSNR and SNR value. This paper makes the fused image is informative and clear from input images. Also, this paper keeps on the details of the edges. It has resulted in the PSNR value of 1 level of dwt is larger than 2level of dwt because increasing the number of levels cause losing the necessary information of images. The fusion technique in this paper applies to more than two images because the PSNR and SNR value of one image is equal to the PSNR and SNR value of another image.

\section{REFERENCES}

[1] Tawfiq A. Abbas and Firas Sabar Miften, "Fractal recognition by using wavelet transform," Journal of Babylon University, vol. 20, no. 6, 2012.

[2] Al-Aboosi, Yasin Yousif, Radhi Sehen Issa, and Ali Khalid Jassim, "Image denoising in underwater acoustic noise using discrete wavelet transform with different noise level estimation." TelkomnikaTelecommunication, Computing, Electronics, and Control, vol. 18, no. 3,pp. 1441-1442, 2020.

[3] Israa Hadi Ali and Russell H. Al_taie, "Wavelet coefficient fusion method -based image denoising", Research Journal of Applied Sciences, vol. 11, no. 10, pp. 1045-1049, 2016.

[4] Pratama, Muhammad Biyan, et al., "Hand gesture recognition using discrete wavelet transform and convolution neural network," Bulletin of Electrical Engineering and Informatics, vol. 9, no. 3, pp. 996-999, 2020, DOI: 10.11591/eei.v9i3.1977.

[5] Israa Hadi Ali and Russell H. Al_taie, "Image deblurring using fusion technique based on inverse filtering," Research Journal of Applied Sciences, vol. 11, no. 11, pp. 1206-1210, 2016.

[6] Israa Hadi Ali and Russell H. Al_taie, "Wavelet coefficient fusion method -based image denoising," Research Journal of Applied Sciences, vol. 10, no. 11, pp. 1045-1049, 2016.

[7] Achmamad, Abdelouahad, and Atman Jbari, "A comparative study of wavelet families for electromyography signal classification based on discrete wavelet transform," Bulletin of Electrical Engineering and Informatics, vol. 9, no.4, pp. 1420-1425, 2020, DOI: 10.11591/eei.v9i4.2381.

[8] Shriniwas T. Budhewar, "Wavelet and Curvelet Transform based Image Fusion Algorithm," International Journal of Computer Science and Information Technologies, vol. 3, no. 5, pp. 3703-3705, 2014.

[9] Setyono, A., and D. R. I. M. Setiadi," Image watermarking using discrete wavelet transform," Indones. J. Electr. Eng. Comput. Sci., vol. 16, no. 3, pp. 1418-1419, 2019.

[10] Singh, Er.Simar Preet and Er. Palak Sharma, "Image Fusion," International Journal of Advanced Research in Computer Science and Software Engineering, vol. 3, no. 4, pp. 206, 2014.

[11] Mamta Sharma, "A Review: Image Fusion Techniques and Applications," International Journal of Computer Science and Information Technologies, vol. 7, no. 3, pp. 1082-1085, 2016.

[12] Ramya, H. R., and B. K. Sujatha, "Fine-grained medical image fusion using type-2 fuzzy logic," Indonesian Journal of Electrical Engineering and Computer Science, vol. 14,no. 2,pp. 999-1004, 2019.

[13] Devyani P. Deshmukh and A. V. Malviya, "Image Fusion an Application of Digital Image Processing using Wavelet Transform," International Journal of Scientific \& Engineering Research, vol. 6, no. 11, pp. 1247-1250, 2015.

[14] Vinay Sahu \& Dinesh Sahu, "Image Fusion using Wavelet Transform: A Review," Global Journal of Computer Science and Technology: FGraphics \& Vision, vol. 14, no. 5, 2014.

[15] Hari, Mishra and Smriti, "MRI and CT Image Fusion Based on Wavelet Transform," International Journal of Information and Computation Technology, vol. 4, no. 1, 2014.

[16] Laxman, Tawade, Aboobacker, and Ghante, “Image Fusion Based on Wavelet Transforms,” International Journal of Bio-Science and Bio-Technology, vol. 6, no. 3, 2014.

[17] Indira, "Image Fusion for Pet Ct Images Using Average Maximum and Average Contrast Rules," International Journal of Applied Engineering Research, vol. 10, no. 1, 2015.

[18] Ram Nivas Singh Yadav and Himanshu Agarwal, "Medical Image Fusion Using Wavelet Transform," International Journal of Scientific Engineering and Applied Science (IJSEAS), vol. 1, no.3, 2015.

[19] Prerana G Agarkar and Prof. Deepali R Sale, "Efficient MRI and CT Images Fusion Technique: Analysis," International Journal of Advanced Research in Computer Science and Software Engineering, vol. 5, no. 7, pp. 594599, 2015.

[20] Nayera Nahvi and Deep Mittal, "Medical Image Fusion Using Discrete Wavelet Transform," Int. Journal of Engineering Research and Applications, vol. 4, no. 9, pp. 165-170, 2014.

[21] Russell Haidar Jassim, Image Denoising Using Fusion Technique Based on DWT Coefficient. MSc, Thesis. College of Information Technology, University of Babylon, 2017.

[22] Priya D. Vora and Ms. Neeta Chudasama, "Different Image Fusion Techniques and Parameters: A Review," International Journal of Computer Science and Information Technologies, vol. 1, no. 6, pp. 889-891, 2015.

[23] Yufeng Zheng, 2011. Image fusion and its applications, InTech Janeza Trdine 9, 51000 Rijeka, Croatia.

[24] Mandhare, Rohan Ashok, Pragati Upadhyay, and Sudha Gupta, "Pixel Level Image Fusion Using Brovey Transform and Wavelet Transform," International Journal of Advanced Research in Electrical Electronics and Instrumentation Engineering, vol. 2, no. 6, 2013. 
[25] A H Russell, "Noise removal from medical image using fusion technique based on DWT coefficient," IOP Conf. Series: Journal of Physics: Conf. Series, pp. 1294, 2019, doi:10.1088/1742-6596/1294/2/022015.

[26] Roa'a, M. Al_airaji, et al., "Generation of High Dynamic Range for Enhancing the Panorama Environment," Bulletin of Electrical Engineering and Informatics, vol. 10, no. 1, pp. 138-147, 2021.

[27] Rusul H. Al_taie, "Restoration for Blurred Noisy Images Based on Guided Filtering and Inverse Filter," International Journal of Electrical and Computer Engineering, vol. 11, no. 2, pp. 1265-1275, 2021.

\section{BIOGRAPHIES OF AUTHORS}
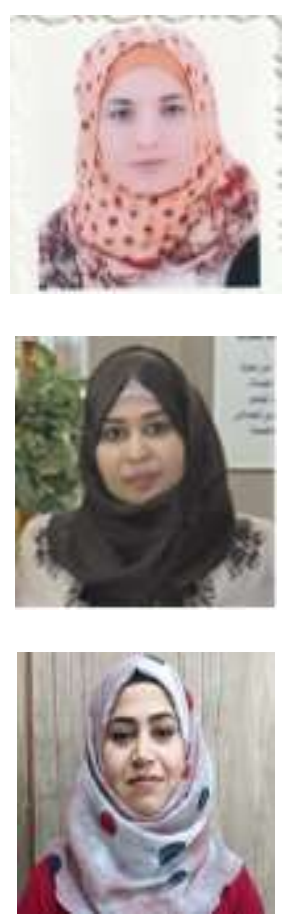

Zahraa Yaseen Hasan is born in Iraq in 1984. I obtained a bachelor's in computer science in 2006. She received her master's Department of Software from the University of Babylon in Iraq, in 2017. Post address: lecturer, University of Babylon, Hilla, Iraq.

Rusul H. Al_taie is born in Iraq in 1988. I obtained a bachelor's in computer science in 2010. She received her master's Department of Software from the University of Babylon in Iraq, in 2017. Her fields of interest include primarily image processing, computer graphics, and data compression.

Hawraa abd Al_kadum hassan is born in Iraq in 1988. I obtained a bachelor's in computer science in 2010. She received her master's Department of Software from the University of Babylon in Iraq in 2017. 